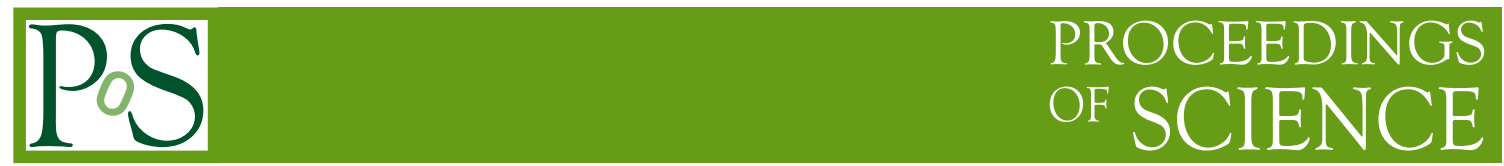

\title{
A novel spectrometer for neutrino experiments
}

\section{Laura Pasqualini*, on behalf of the NESSiE Collaboration}

University of Bologna and INFN

E-mail: laura.pasqualiniabo.infn.it

The WA104-NESSiE program developed in the context of the CERN Neutrino Platform, includes an innovative spectrometer to measure the charge and the momentum of muons in $0.5-5 \mathrm{GeV} / \mathrm{c}$ range. A tracking apparatus with a spatial resolution of $1 \mathrm{~mm}$ was designed, to be placed in a magnetized air volume in order to achieve a charge resolution and mis-identification of better than $1 \%$ at $1 \mathrm{GeV} / \mathrm{c}$. Preliminary results obtained by detecting cosmic ray muons are reported.

XVI International Workshop on Neutrino Telescopes,

2-6 March 2015

Palazzo Franchetti - Istituto Veneto, Venice, Italy

${ }^{*}$ Speaker. 


\section{Tracking system}

The WA104-NESSiE program includes the development of a novel spectrometer for applications in future neutrino experiments. Preliminary studies have shown that a tracking system with a spatial resolution of about $1 \mathrm{~mm}$, operating inside a $0.12 \mathrm{~T}$ magnetized air volume (Air Core Magnet) would allow a charge mis-identification of about $1 \%$ at $1 \mathrm{GeV} / \mathrm{c}$, required for an accurate determination of the neutrino beam components [1,2].

The Air Core Magnet (see figure 1 (a)) is made of Aluminium coils, $72 \times 72 \mathrm{~mm}^{2}$ cross section, with a circulating $2 \mathrm{~A} / \mathrm{mm}^{2}$ current density generating a $0.12 \mathrm{~T}$ magnetic field in an air volume 1.3 $m$ deep.

The tracking detector is made of planes of triangular scintillator bars (Figure 1 (b)) equipped with Silicon Photomultipliers (SiPMs) read in analog mode. In preliminary tests it was used a tracking prototype composed of 2 modules of triangular bars (each module is a 2-plane system, 4 channels each). The scintillator bars are $3.3 \times 1.7 \times 50 \mathrm{~cm}^{3}$ with embedded WaveLenght Shifting (WLS) fibers (Figure 1 (c)).

A DAQ system based on NIM and VME standard was setup. Scintillators signals are acquired in analog mode by 16 channels $250 \mathrm{MS} / \mathrm{s}$ waveform digitizer.

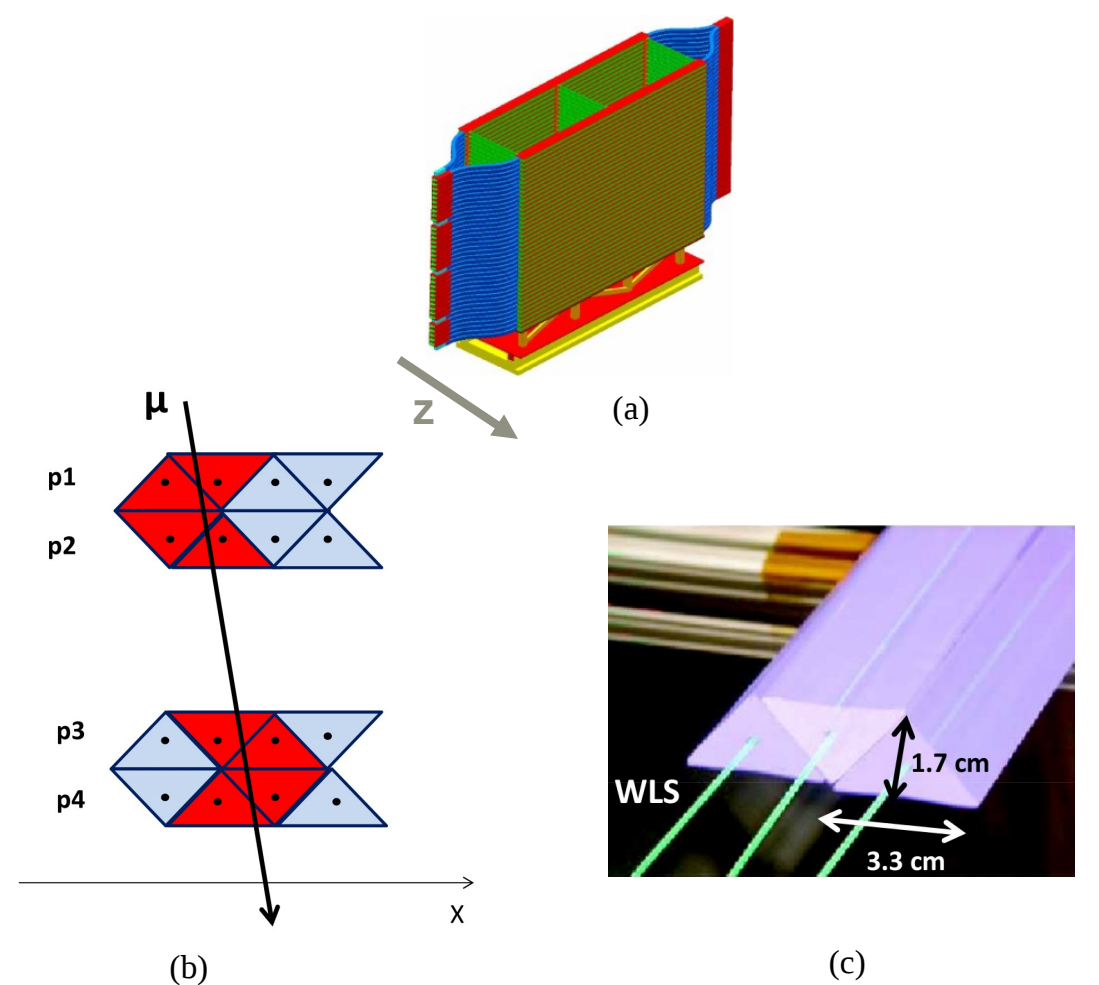

Figure 1: (a) Design of the Air Core Magnet spectrometer. (b) Sketch of the R \& D layout. The tracking system used for tests is composed by 2 modules of triangular scintillator bars. Each module has 2 detector planes, 4 channels each. Red triangular bars have a signal due to the passage of a cosmic ray muon. (c) Picture (adapted from Ref. [5]) of scintillator bars with embedded WLS. 
Features of SiPM like single photon detection, reduced size, low power consumption make them particularly suitable for achieving a low-cost performing tracking device [3]. Moreover being insensitive to magnetic field [4] they represent a natural choice for a detector to be placed inside the ACM of WA104.

SiPM SenSL-10035 device used for preliminary tests has $1 \times 1 \mathrm{~mm}^{2}$ active area with 504 microcells (64\% fill factor) and a gain factor of $10^{6}$ photoelectrons. The main source of noise which limits the SiPM's single photon resolution is the dark current rate. Dark current is originated from charge carriers thermally created in the sensitive volume and present in the conduction band; therefore it is dependent from the temperature. The dark current rate including other kind of noise (i.e. cross talk and after pulses) is $\sim 900 \mathrm{kHz}$, with a cross talk probability (defined as the ratio between 2-cells and 1-cell rate) of about $15 \%$. The variation of the dark current in the typical range of laboratory temperatures $\left(15-28{ }^{\circ} \mathrm{C}\right)$ is $1.8 \%{ }^{\circ} \mathrm{C}$.

The position $X_{\text {reco }}$ of a particle crossing a plane of triangular bars is reconstructed from the pulse height in each channel, namely:

$$
X_{\text {reco }}=\frac{\sum_{i} w_{i} x_{i}}{\sum_{i} w_{i}}
$$

where $x_{i}$ is the fiber nominal position and $w_{i}$ is the pulse height in the i-th channel, proportional to the energy lost by the particle in the bar. Only tracks that leave a signal in one channel and in its adjacent ones are reconstructed.

\section{Data analysis}

Tests triggering on cosmic rays muons were performed requiring at least a signal over threshold in each plane of triangular bars. A threshold on each channel was set to about 5 pixel (50-55

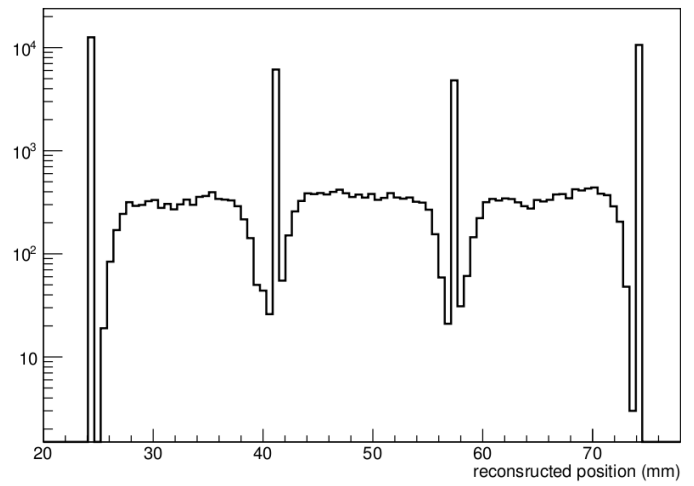

(a)

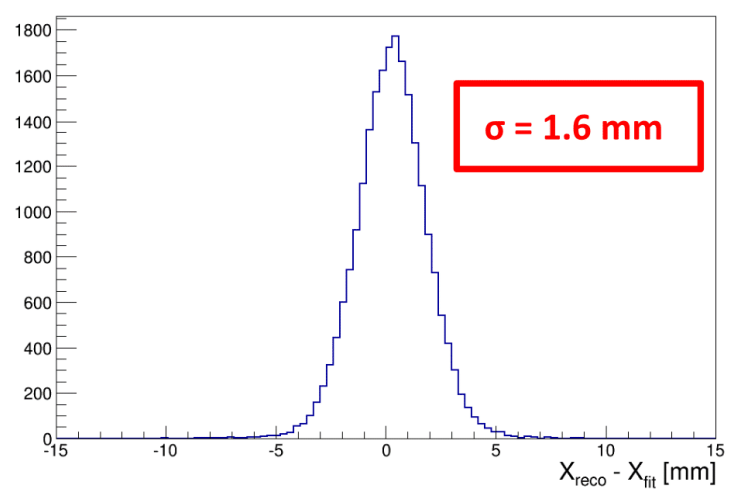

(b)

Figure 2: (a) Reconstructed positions in a plane of triangular bars. A software cut of $30 \mathrm{mV}$ was applied to each signal. (b) Residuals between the reconstructed $X_{\text {reco }}$ positions and fitted track projections $X_{f i t}$ in each plane of triangular bars for events with only 2 adjacent triangular bars fired in each plane. The achieved spatial resolution is better than $2 \mathrm{~mm}$. 
$\mathrm{mV})$. The triangular bar efficiency is about $95 \%$. Before data taking, the response of each bars coupled with a SiPM was tested by laser injecting light pulses. Under these requirements a total of about $260 \times 10^{3}$ events was collected at a rate of $1.7 \mathrm{~Hz}$.

The reconstructed $X_{\text {reco }}$ positions of CR events were evaluated for each plane. In figure 2 (a) is shown the distribution of reconstructed positions in a plane of triangular bars. A software cut of $30 \mathrm{mV}$ was applied to each signal. Bins with high statistics correspond to events with only a triangular bar fired in that plane, which cannot have the position reconstructed by the formula 1.1. A nominal position is therefore assigned. A linear fit of the reconstructed positiond in each plane was performed. Events with only 2 adjacent triangular bars fired in each plane are selected. Residuals between the reconstructed positions and the projected positions of fitted track in each plane are shown in figure 2 (b). The RMS obtained is better than $2 \mathrm{~mm}$.

A geometrical simulation of the tracking detector was implemented showing that the spatial resolution in reconstructing tracks is about $1.1 \mathrm{~mm}$.

\section{Conclusions}

A system made of planes of triangular scintillator bars equipped with SiPM read in analog mode was developed for the reconstruction of low energy $(\mathrm{O}(1 \mathrm{GeV}))$ muon tracks. Tests with cosmic ray muons have shown that a spatial resolution better than $2 \mathrm{~mm}$ can be achieved.

This experimental approach will be implemented in a multiplane (meter scale, with $\sim 10$ planes) prototype to be tested with charged beams in order to set the ultimate achievable spatial accuracy.

\section{References}

[1] NESSiE Collaboration, P. Bernardini et al., SPSC-P-343 (2011), arXiv:1111.2242.

[2] NESSiE Collaboration, A. Anokhina et al., CERN-SPSC-2014-007.

[3] Dolgoshein B. et al., NIM A563 (2006) 368.

[4] Berra A. et al., NIM A609 (2009) 129.

[5] arXiv:1305.5199 [physics.ins-det]. 